\title{
Genoepidemiological Evaluation of Bovine viral diarrhea virus 2 Species Based on Secondary Structures in the 5' Untranslated Region
}

\author{
Massimo GIANGASPERO ${ }^{1) *}$, Ryô HARASAWA ${ }^{1)}$, Laura WEBER ${ }^{2)}$ and Angelo BELLOLI ${ }^{3)}$ \\ ${ }^{1)}$ Department of Veterinary Microbiology, Faculty of Agriculture, Iwate University, Morioka 020-8550, Japan, ${ }^{2)}$ Instituto de Virologia, \\ Centro de Investigacion en Ciencias Veterinarias y Agronomicas (CICVyA), INTA-Castelar, CC25 (1712), Castelar, Buenos Aires, \\ Argentina and ${ }^{3)}$ Department of Veterinary Medical Sciences, The University of Milan, via Celoria 10, 20133 Milan, Italy
}

(Received 15 February 2007/Accepted 7 February 2008)

\begin{abstract}
Bovine viral diarrhea virus 2 (BVDV-2) strains demonstrated in cattle, sheep, and adventitious contaminants of biological products have been evaluated by the palindromic nucleotide substitutions (PNS) method at the three variable loci (V1, V2 and V3) in the 5' untranslated region (UTR), to determine their taxonomical status. Variation in conserved genomic sequences was used as parameter for epidemiological evaluation of the species in relation with geographical distribution, animal host and virulence. Four genotypes, BVDV-2a, BVDV-2b, BVDV-2c, and BVDV-2d have been identified within the species. Taxonomical segregation corresponded to geographical distribution of genotype variants. Genotype 2 a was present worldwide, and was the only circulating also in sheep, in addition to cattle. Genotypes 2b, $2 \mathrm{c}$ and $2 \mathrm{~d}$ were restricted to South America. Contamination of biological products was related to genotypes $2 \mathrm{a}$ and $2 \mathrm{~d}$. Genetic variation could be related with chronological diffusion of the BVDV-2 species variants in different geographic areas. Chronologically, the species emerged in North America in 1978, spreading in UK and Japan, continental Europe, South America and New Zealand. Correlation between clinical features related with isolation of BVDV-2 strains and genetic variation indicated that subgenotype 1, variant 4 of genotype 2a was related with hemorrhagic syndrome. These observations suggest that evaluation of genomic secondary structure, by identifying markers for expression of virus biological activities and species evolutionary history, may be applied as useful tool for epidemiological evaluation of the BVDV-2 species, and possibly for other species of the genus Pestivirus. KEY WORDS: BVDV-2, genoepidemiology, palindromic nucleotide substitutions, pestivirus.
\end{abstract}

Bovine viral diarrhea virus 2 (BVDV-2), represents an established species of the genus Pestivirus of the family Flaviviridae, with Bovine viral diarrhea virus 1 (BVDV-1), Border disease virus (BDV) and Classical swine fever virus (CSFV) and a tentative "Giraffe" species [12]. BVDV-2 species includes the strains isolated from outbreaks hemorrhagic syndrome characterised by thrombocytopenia and high mortality in U.S.A. and Canada [5, 25, 27]. Hyper-virulent strains 890 and CD-87 have been previously included in BVDV species in a separate cluster [25, 28]. The hemorrhagic syndrome has been reported also in European cattle $[7,24,36]$. Different strains were also isolated in bovine animals showing mild clinical forms $[22,35]$. Furthermore, the presence of the species was observed in biological products as adventitious contaminant $[4,16,18,20]$, indicating the risk of iatrogenic infection, demonstrated by the recent occurrence of a serious accident in the Netherlands among cattle, who received a live virus vaccine contaminated with pestivirus [11].

The Pestivirus genome, single-stranded, positive polarity RNA, is composed by a sequence of about 12,500 nucleotides, which can be divided into three regions: a 5'-untranslated region (UTR) containing an internal ribosomial entry site (IRES), a single large open reading frame encoding a polyprotein, and a 3'-UTR. The 5'-UTR is highly con-

\footnotetext{
* Correspondence to: Giangaspero, M., Department of Veterinary Microbiology, Faculty of Agriculture, Iwate University, Morioka 020-8550, Japan.

e-mail: giangasp@iwate-u.ac.jp
}

served among all members within the genus Pestivirus, thus being useful for the characterization of species or genotypes. The primary structure analysis, by sequence alignment and construction of phylogenetic trees, is the most common method for the classification of the Pestivirus isolates. However, it is relatively easy to predict the secondary structure, according to the most probable nucleotide binding, with lowest folding energies. The secondary structure of the 5'-UTR can be divided into four domains, A-D, with domain D encompassing the two thirds in the 3' region of the 5'-UTR predicted to fold into a complex palindromic stem-loop structure $[9,16]$, a critical region of the 5'-UTR, which is responsible for translational, transcriptional and replicational events in pestiviruses. Therefore, random mutations at the 5'-UTR have a high probability of incompatibility with viral survival. Thus stable nucleotide variations at this level assume high importance in terms of virus evolutionary history. The nucleotide substitutions of the IRES in the 5'-UTR were supposed to be responsible for the virulence of poliovirus [26]. Mutations or nucleotide substitutions at palindromic IRES regions will affect efficiency translation from RNA to protein. Thus low translation might reduce virulence, and high translation increase virulence. Due to genetic structure similarity with pestiviruses, this concept was proposed for BVDV-2 by Topliff and Kelling [35]. The authors reported a relatedness between high virulence and two specific nucleotides, uracil and cytosine located at position 219 and 278 of the genomic sequence (based on BVDV-1 strain Osloss) and low viru- 
lence with cytosine and uracil at position 219 and 278, respectively. Nucleotide sequences at the three variable loci, V1, V2 and V3, in the 5'-UTR of pestiviruses have been shown to be palindromic and capable of forming a stable stem-loop structure peculiar to each Pestivirus species. Nucleotide substitutions in the stem regions always occur to maintain the palindromic sequence and thereby form a stable stem-loop structure. Thus, this type of mutation was referred to palindromic nucleotide substitutions (PNS). Based on the above mentioned considerations, the observation of nucleotide variations among virus strains at the level of the three specific palindromes in the 5'-UTR has been conceived as method for genotyping [17]. The method named PNS analysis appeared to be simple and practical, showing comparable results with other procedures based on the primary structure comparison. The results of the PNS method can be essentially qualitative and provide the exact species classification of an isolate. Thus, this method may help to clarify species and genotype boundaries, due to the exclusive consideration of strategic and highly conserved regions, and consequently helps to avoid unclear classification.
In the present study, BVDV-2 Pestivirus strains, isolates from cattle and sheep, and adventitious contaminants of biological products, have been evaluated with PNS analysis in the 5'-UTR to determine relation between genotypic variations and epidemiological observations.

\section{MATERIALS AND METHODS}

The nucleotide sequences in the 5'-UTR of seventy-three BVDV-2 species strains were obtained from the DNA databases or provided directly from the authors via personal communication (sequences not deposited by Beer, Vilcek and Ridpath; sequences defined as ALIGN_000012 by Couvreur and available on the EMBL web site) (Table 1). According to the geographic origin of the virus isolates fifteen were reported from North America, and twelve from South America, thirty two from Europe, thirteen from Japan, and one from New Zealand. Eight strains originated from sheep, isolated from outbreaks of border disease. Twelve strains were detected as contaminats from cell cultures and biologicals. The strain IT-1732 (AJ416018) (Muscillo, unpublished) was isolated from a bayovac IBR-

Table 1. BVDV-2 strains evaluated according to secondary structure nucleotide chages. ND: not determined

\begin{tabular}{|c|c|c|c|c|c|c|c|}
\hline Genotype & Strain & Origin & Country & Year of isolation & Clinical presentation & Accession & Reference \\
\hline BVDV-2a1.1 & $5521-95$ & Cattle & USA & 1995 & Fetal abortion & AF039174 & Topliff \& Kelling,98 \\
\hline BVDV-2a1.1 & $713-2$ & Cattle & USA & 1982 & Fetal abortion & AF039177 & Topliff \& Kelling,98 \\
\hline BVDV-2a1.1 & $97 / 730$ & Cattle & NewZealand & dd 1997 & Data not availables & AF026770 & Vilcek et al., unpublished \\
\hline BVDV-2a1.1 & BSE1239 & Cattle & Belgium & 1996 & Neurological symptoms & ALIGN_000012 & Couvreur et al., 02 \\
\hline BVDV-2a1.1 & UVR420 & Cattle & Belgium & 1991 & Calf intestine & ALIGN_000012 & Couvreur et al., 02 \\
\hline BVDV-2a1.2 & BD-78 & Sheep & USA & 1978 & Border disease & U18330 & Sullivan et al., 94 \\
\hline BVDV-2a1.2 & $\mathrm{C} 413$ & Sheep & USA & 2002 & Border disease & AF002227 & Chen \& Berry, unpublished \\
\hline BVDV-2a1.3 & 167237 & Sheep & UK & 1987 & Border disease & U65055 & Vilcek et al., 97 \\
\hline BVDV-2a1.3 & 168149 & Sheep & UK & 1987 & Border disease & U65056 & Vilcek et al., 97 \\
\hline BVDV-2a1.3 & 173157 & Sheep & UK & 1987 & Border disease & U65058 & Vilcek et al., 97 \\
\hline BVDV-2a1.3 & 175375 & Sheep & UK & 1987 & Border disease & U65059 & Vilcček et al., 97 \\
\hline BVDV-2a1.3 & BSE921 & Cattle & Belgium & 1995 & Neurological symptoms & ALIGN_000012 & Couvreur et al., 02 \\
\hline BVDV-2a1.3 & Lees & Sheep & UK & 1985 & Border disease & U65051 & Vilcek et al., 97 \\
\hline BVDV-2a1.3 & $\mathrm{CPA}$ & Contaminant & Japan & 1993 & Not applicable & D50812 & Harasawa \& Mizusawa, 95 \\
\hline BVDV-2a1.3 & CPAE & Contaminant & Japan & 1993 & Not applicable & D50813 & Harasawa \& Mizusawa, 95 \\
\hline BVDV-2a1.3 & EBTr & Contaminant & Japan & 1993 & Not applicable & D50817 & Harasawa et al., 93 \\
\hline BVDV-2a1.3 & HE727 & Contaminant & Japan & 1995 & Not applicable & D31807 & Harasawa \& Mizusawa, 95 \\
\hline BVDV-2a1.3 & MMR-T & Contaminant & Japan & 1993 & Not applicable & D26052 & Harasawa \& Tomiyama, 94 \\
\hline BVDV-2a1.3 & MP & Contaminant & Belgium & 2002 & Not applicable & ALIGN_000012 & Couvreur et al., 02 \\
\hline BVDV-2a1.3 & Parvo & Contaminant & Japan & 1994 & Not applicable & D26614 & Harasawa, 94 \\
\hline BVDV-2a1.3 & Rubella & Contaminant & Japan & 1993 & Not applicable & D26048 & Harasawa \& Tomiyama, 94 \\
\hline BVDV-2a1.4 & 11/Mi/97 & Cattle & Italy & 1997 & Data not availables & AJ293603 & Luzzago et al., 00 \\
\hline BVDV-2a1.4 & $15-103$ & Cattle & France & 1994 & Data not availables & AF298055 & Vilcek et al., 01 \\
\hline BVDV-2a1.4 & $17583-97$ & Cattle & USA & 1997 & Acute BVD & AF039176 & Topliff \& Kelling,98 \\
\hline BVDV-2a1.4 & 23025 & Cattle & USA & 1993 & Hemorrhagic syndrome & AF039172 & Topliff \& Kelling,98 \\
\hline BVDV-2a1.4 & $37 \mathrm{Gr}$ & Cattle & Austria & $98-00$ & Acute diarrhea & not deposited & Vilcek et al., 03 \\
\hline BVDV-2a1.4 & 7937 & Cattle & USA & 1988 & Persistent infection & AF039175 & Topliff \& Kelling,98 \\
\hline BVDV-2a1.4 & AZ Spl & Cattle & USA & $60-93$ & Hemorrhagic syndrome & not deposited & Ridpath et al., 94 \\
\hline BVDV-2a1.4 & BSE341 & Cattle & Belgium & 1991 & Neurological symptoms & ALIGN_000012 & Couvreur et al., 02 \\
\hline BVDV-2a1.4 & IT-1732 & Contaminant & Italy & 1999 & Acute BVD & AJ416018 & Muscillo, unpublished \\
\hline BVDV-2a1.4 & Kosice & Cattle & Slovakia & 2002 & $\begin{array}{l}\text { Fatal course } \\
\text { Malformations, } \\
\text { hemorrhagies }\end{array}$ & not deposited & Vilcek et al., 02 \\
\hline BVDV-2a1.4 & MAD Spl & Cattle & USA & $60-93$ & Hemorrhagic syndrome & not deposited & Ridpath et al., 94 \\
\hline BVDV-2a1.4 & MN Fetus & Cattle & USA & $60-93$ & Hemorrhagic syndrome & not deposited & Ridpath et al., 94 \\
\hline
\end{tabular}


Marker vaccine (Bayer), responsible for outbreaks in the Netherlands and Italy [11]. The strain WG4622 [ALIGN_000012] [8] was isolated from the same batch of vaccine. Strains MMR-T [D26052], Rubella [D26048] [16, 20] and HE727 [D31807] [19], were isolated from Japanese commercial virus vaccines and interferons for human use. Strain 354 [AF244959] [21] was isolated in Argentina.

The secondary structures were predicted according to the algorithm of Zuker and Stiegler [40]. The minimum free energy was calculated by the method of Freier et al. [14]. Three variable regions, V1, V2 and V3, at the 5'-UTR were used for genotyping based on palindromic nucleotide substitutions [17]. In each variable locus, nucleotide base pairs have been defined as positions starting their numbering from the bottom of the stems of the palindromes. Sequences were aligned according to secondary structure to evaluate nucleotide changes in relation with geographic distribution, animal hosts and virulence, identifying specific PNS markers.

\section{RESULTS}

The evaluation of the BVDV-2 Pestivirus strain 5'-UTR nucleotide sequences, according to PNS analysis allowed the identification of specific nucleotide changes among genotype variants of the species, related with geographic segregation, as well as animal host origin and virulence.

Characteristic base pairings common to BVDV-2 species were observed in all tested isolates. The application of the PNS method showed four genotypes, BVDV-2a, BVDV-2b,

Table 1. (cont'd)

\begin{tabular}{|c|c|c|c|c|c|c|c|}
\hline Species & Strain & Origin & Country & Year of isolation & Clinical presentation & Accession & Reference \\
\hline BVDV-2a1.4 & NY93 & Cattle & USA & 1993 & Acute BDV & AF039173 & Topliff \& Kelling,98 \\
\hline BVDV-2a1.4 & Q126 & Cattle & Canada & 1994 & Hemorrhagic syndrome & L32890 & Pellerin et al., 94 \\
\hline BVDV-2a1.4 & V-FLL & Contaminant & Japan & 1999 & Not applicable & AB019687 & Sakoda et al., 99 \\
\hline BVDV-2a1.4 & WG4622 & Contaminant & Netherland & 1999 & Letal acute BVD & ALIGN 000012 & Couvreur et al., 02 \\
\hline BVDV-2a1.4 & WVD829 & Cattle & Belgium & 1994 & Respiratory symptoms & ALIGN_000012 & Couvreur et al., 02 \\
\hline BVDV-2a1.5 & 890 & Cattle & Canada & 1990 & Hemorrhagic syndrome & L32886 & Pellerin et al., 94 \\
\hline BVDV-2a1.6 & $104 / 98$ & Cattle & Germany & 1998 & Mild symptoms & AJ304381 & Tajima et al., 01 \\
\hline BVDV-2a1.6 & B52-2 & Cattle & Germany & $92-99$ & Mild symptoms & not deposited & Beer et al., 02 \\
\hline BVDV-2a1.6 & CD87 & Cattle & Canada & 1987 & Hemorrhagic syndrome & L32887 & Pellerin et al., 94 \\
\hline BVDV-2a1.6 & i4083 & Cattle & Argentina & 2001 & Mild symptoms & AF417995 & Jones et al., unpublished \\
\hline BVDV-2a1.6 & i61380 & Cattle & Argentina & 2001 & Mild symptoms & AF417986 & Jones et al., unpublished \\
\hline BVDV-2a1.6 & $\mathrm{i} 628$ & Cattle & Argentina & 2001 & Mild symptoms & AF417985 & Jones et al., unpublished \\
\hline BVDV-2a1.6 & Munich 1 & Cattle & Germany & $92-99$ & Mild symptoms & not deposited & Beer et al., 02 \\
\hline BVDV-2a1.6 & Munich 2 & Cattle & Germany & $92-99$ & Mild symptoms & not deposited & Beer et al., 02 \\
\hline BVDV-2a1.6 & $4-5174$ & Cattle & France & 1994 & Data not availables & AF298063 & Vilček et al., 01 \\
\hline BVDV-2a2 & B45-5 & Cattle & Germany & $92-99$ & Mild symptoms & not deposited & Beer et al., 02 \\
\hline BVDV-2a2 & B50-5 & Cattle & Germany & $92-99$ & Mild symptoms & not deposited & Beer et al., 02 \\
\hline BVDV-2a2 & B5-4 & Cattle & Germany & $92-99$ & Mild symptoms & not deposited & Beer et al., 02 \\
\hline BVDV-2a2 & B77-5 & Cattle & Germany & $92-99$ & Mild symptoms & not deposited & Beer et al., 02 \\
\hline BVDV-2a2 & Giessen-1 & Cattle & Germany & 1996 & Fatal mucosal disease & AF 104030 & Becher et al., 99 \\
\hline BVDV-2a2 & Munich 3 & Cattle & Germany & $92-99$ & Mild symptoms & not deposited & Beer et al., 02 \\
\hline BVDV-2a2 & 17011-96 & Cattle & USA & 1996 & Fetal abortion & AF039179 & Topliff \& Kelling, 98 \\
\hline BVDV-2a2 & AF112 & Cattle & Germany & $92-99$ & Mild symptoms & not deposited & Beer et al., 02 \\
\hline BVDV-2a2 & BS-95-II & Cattle & Italy & 1995 & Data not availables & AJ288903 & Luzzago et al., 00 \\
\hline BVDV-2a2 & MS-1 & Cattle & Japan & 1999 & Persistent infection & AB019688 & Sakoda et al., 99 \\
\hline BVDV-2a2 & SW90 & Cattle & Japan & 1990 & Mild symptoms & $\mathrm{AB} 003622$ & Nagai et al., 01 \\
\hline BVDV-2a2 & SY-89 & Cattle & Japan & 1989 & Persistent infection & AB019689 & Sakoda et al., 99 \\
\hline BVDV-2a2 & OY89 & Cattle & Japan & 1989 & $\begin{array}{l}\text { Persistent infection } \\
\text { Respiratory symptoms }\end{array}$ & $\mathrm{AB} 003621$ & Nagai et al., 98 \\
\hline BVDV-2a2 & TC Shinozaki & Cattle & Japan & 1992 & $\begin{array}{l}\text { Persistent infection } \\
\text { Respiratory symptoms }\end{array}$ & AB04267 & Nagai et al., 01 \\
\hline BVDV-2b1.1 & VS-63 & Cattle & Brazil & 1996 & Healthy fetus & AF410789 & Flores et al., 02 \\
\hline BVDV-2b1.2 & $34 \mathrm{~b}$ & Cattle & Argentina & 1991 & Fetus & AF244952 & Jones et al., 01 \\
\hline BVDV-2b1.2 & ncp7 & Cattle & Argentina & 1993 & Fetus & AY443026 & Jones et al., 01 \\
\hline BVDV-2b1.3 & VS-123.4 & Cattle & Brazil & 1996 & Healthy fetus & AF410790 & Flores et al., 02 \\
\hline BVDV-2b1.4 & LV-96 & Cattle & Brazil & 1996 & Chronic gastroenteric & AF410787 & Flores et al., 02 \\
\hline BVDV-2b1.4 & VS-260 & Cattle & Brazil & 1997 & Gastroenteric symptoms & s AF410788 & Flores et al., 02 \\
\hline BVDV-2b2 & Soldan & Cattle & Brazil & 1991 & Mucosal disease & U94914 & Canal et al., unpublished \\
\hline BVDV-2c & $\mathrm{i} 33283$ & Cattle & Argentina & 2001 & Mild symptoms & AF417996 & Jones et al., unpublished \\
\hline BVDV-2d & 354 & Contaminant & Argentina & 1995 & Not applicable & AF244959 & Jones et al., 01 \\
\hline BVDV-2 ND & 59386 & Sheep & UK & 1994 & Border disease & U17146 & Becher et al., 94 \\
\hline BVDV-2 ND & SCP & Contaminant & UK & 1994 & Not applicable & U17148 & Becher et al., 94 \\
\hline
\end{tabular}


Table 2. Geographycal distribution and origin of isolation of BVDV-2 genotypes, subgenotypes and variants * contaminants only; Yes: detected; No: not detected

\begin{tabular}{|c|c|c|c|c|c|c|c|c|}
\hline \multirow{2}{*}{$\begin{array}{l}\text { Genotypes } \\
\text { BVDV-2a }\end{array}$} & \multirow{2}{*}{$\begin{array}{c}\text { Subgenotype } \\
1\end{array}$} & \multirow{2}{*}{$\begin{array}{c}\text { Variant } \\
1\end{array}$} & \multicolumn{2}{|c|}{ North America } & South America & \multirow{2}{*}{$\begin{array}{l}\text { Europe } \\
\text { Belgium }\end{array}$} & \multirow[t]{2}{*}{ Asia } & \multirow{2}{*}{$\begin{array}{l}\text { Austral-asia } \\
\text { NewZealand }\end{array}$} \\
\hline & & & USA & & & & & \\
\hline BVDV-2a & 1 & 2 & USA & & & & & \\
\hline BVDV-2a & 1 & 3 & & & & Belgium, UK & Japan* & \\
\hline BVDV-2a & 1 & 4 & Canada & USA & & Austria, Belgium, & Japan & \\
\hline BVDV-2a & 1 & 5 & Canada & & & & & \\
\hline BVDV-2a & 1 & 6 & Canada & & Argentina & France, Germany & & \\
\hline BVDV-2a & 2 & - & USA & & & Germany, Italy & Japan & \\
\hline BVDV-2b & 1 & 1 & & & Brazil & & & \\
\hline BVDV $-2 b$ & 1 & 2 & & & Argentina & & & \\
\hline BVDV-2b & 1 & 3 & & & Brazil & & & \\
\hline BVDV-2b & 2 & - & & & Brazil & & & \\
\hline BVDV-2c & - & - & & & Argentina & & & \\
\hline BVDV-2d & - & - & & & Argentina* & & & \\
\hline Genotypes & Subgenotype & Variant & Cattle & Sheep & Contaminant & & & \\
\hline BVDV-2a & 1 & 1 & Yes & No & No & & & \\
\hline BVDV-2a & 1 & 2 & No & Yes & No & & & \\
\hline BVDV-2a & 1 & 3 & Yes & Yes & Yes & & & \\
\hline BVDV-2a & 1 & 4 & Yes & No & Yes & & & \\
\hline BVDV-2a & 1 & 5 & Yes & No & No & & & \\
\hline BVDV-2a & 1 & 6 & Yes & No & No & & & \\
\hline BVDV-2a & 2 & - & Yes & No & No & & & \\
\hline BVDV-2b & 1 & 1 & Yes & No & No & & & \\
\hline BVDV-2b & 1 & 2 & Yes & No & No & & & \\
\hline BVDV-2b & 1 & 3 & Yes & No & No & & & \\
\hline BVDV-2b & 2 & - & Yes & No & No & & & \\
\hline BVDV-2c & - & - & Yes & No & No & & & \\
\hline BVDV-2d & - & - & No & No & Yes & & & \\
\hline
\end{tabular}

BVDV-2c, and BVDV-2d. Two strains from Argentina were classified as genotypes BVDV-2c and BVDV-2d, showing unexpected base pair changes, suggesting their intermediate phylogenetic position between North American, European and Asian strains and South American ones. Genotype 2b, 2c and 2d South American strains were characterized by GA or GG or AC in position 17 of V1 locus (V1/17), V1/18 GG or GA and a higher V3 loop. Genotype $2 \mathrm{c}$ shared with $2 \mathrm{~b}$ characteristic pairing GC in $\mathrm{V} 1 / 12$, thus in V1/16 showed CG, common pairing in strains from other continents, instead of UA pairing common to other South American strains. In addition a specific CG pairing in position 14 of V1 characterised the genotype 2c. Genotype 2d shared with $2 \mathrm{~b}$ UA pairing in $\mathrm{V} 1 / 16$, but was divergent in V1/12 showing AU common to strains from North America, Europe or Japan. In addition, AU pairing in position 9 of the $\mathrm{V} 1$ locus was specific to the genotype 2d. Genotype 2a was present worldwide, and was the only circulating also in sheep, in addition to cattle, prevalent host species. Genotypes $2 \mathrm{~b}, 2 \mathrm{c}$ and $2 \mathrm{~d}$ were restricted to South America (Table 2).

Taxonomical segregation corresponded to geographical distribution of genotype variants. Chronologically, the species was first detected in North America in 1978, and further in UK and Japan, thus in continental Europe, South America and New Zealand. Genetic variation could be correlated to chronological detection of the BVDV2 species variants in different geographic areas (Fig. 1). Genotype 2a subgenotype 1 variant 1,4 and 6, first reported in North America in 1982, 1988 and 1987, appeared to circulate worldwide. Variant 1 was reisolated in continental Europe in 1991, thus in New Zaland in 1997. Variant 4, mainly highly virulent in cattle, was reported in Europe in 1991 showing mild clinical symptoms, and in 1998-00 related with high virulence. The variant was further reported in Japan as contaminant in 1999. Variant 6, related with hemorrhagic syndrome in Canada, was reisolated in Germany in 1994, and in Argentina in 2001, associated with mild symptoms. Interestingly, subgenotype 2 was first reported in Japanese cattle, and further reisolated in 1995 in Europe and in 1996 in U.S.A. Despite sequence similarities and identities, generated by animal trade, specific nucleotide changes, especially at the level of V1 and V3 loops, allowed the distinguishing of strains originated from North and South America, continen- 
North America

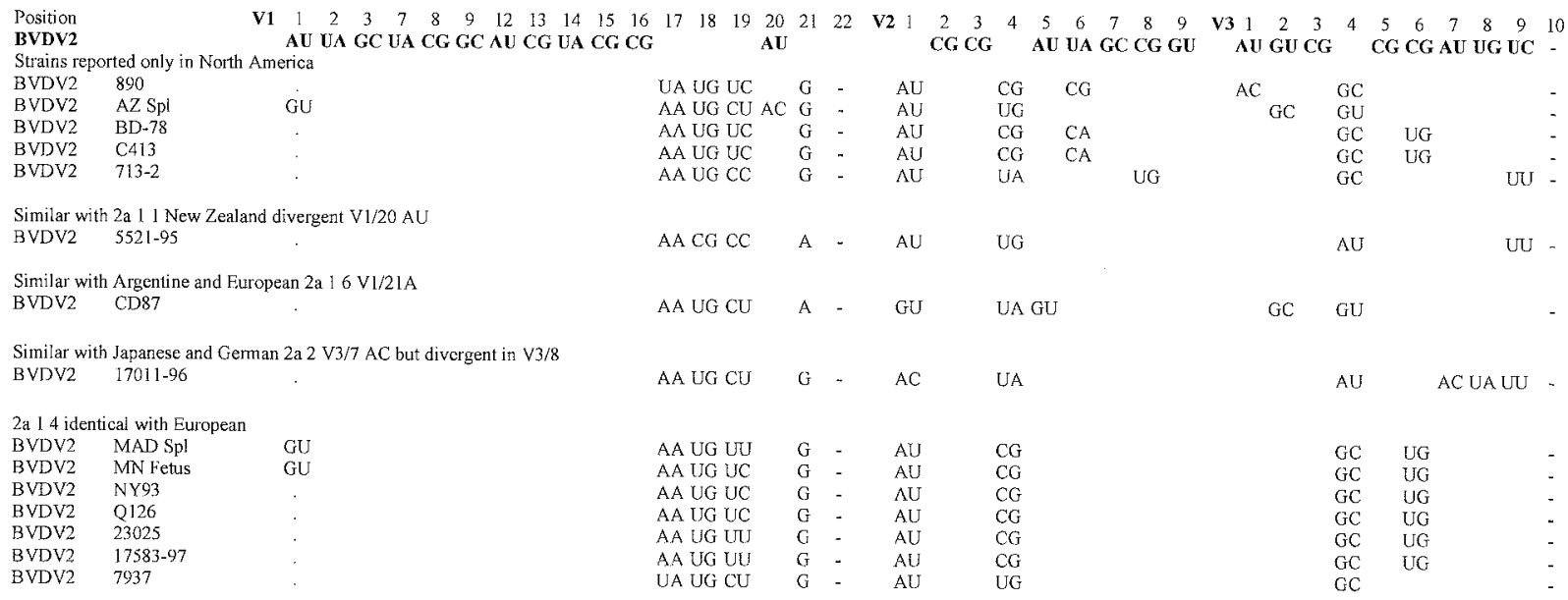

\section{South America}

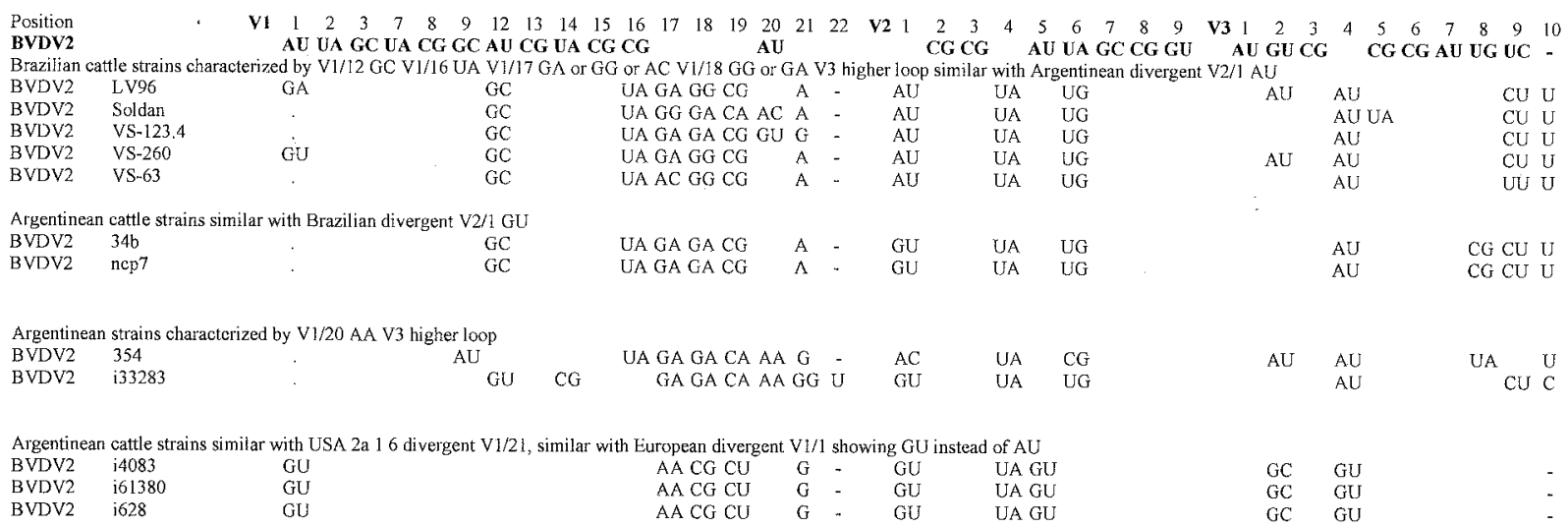

\section{Japan}

$\begin{array}{lllllllllllllllllllllllllllllllllllllllllll}\begin{array}{l}\text { Position } \\ \text { BVDV2 }\end{array} & \text { V1 } & 1 & 2 & 3 & 7 & 8 & 9 & 12 & 13 & 14 & 15 & 16 & 17 & 18 & 19 & 20 & 21 & 22 & \text { V2 } & 1 & 2 & 3 & 4 & 5 & 6 & 7 & 8 & 9 & \text { V3 } & 1 & 2 & 3 & 4 & 5 & 6 & 7 & 8 & 9 & 10\end{array}$ BVDV2 AU UA GC UA CG GC AU CG UA CG CG
Cattle strains characterized by V3/7 AC V3/8 UG or UU, similar with USA $17011-98$ divergent in V3/8

BVDV2 MS-

$\begin{array}{ll}\text { BVDV2 } & \text { OY89 } \\ \text { BVDV2 } & \text { SW90 }\end{array}$

$\begin{array}{ll}\text { BVDV2 } & \text { SW90 } \\ \text { BVDV2 } & \text { SY-89 }\end{array}$

BVDV2 $\quad$ TC Shinozaki

$\begin{array}{lllll}\text { AU UG CU } & \text { G } & - & \text { AC } & \text { UA } \\ \text { AU UG CU } & \text { G } & - & \text { AC } & \text { UA } \\ \text { AU UG CU } & \text { G } & - & \text { AC } & \text { UA } \\ \text { AU UG CU } & \text { G } & - & \text { AC } & \text { UA } \\ \text { AU UG CU } & \text { G } & - & \text { AC } & \text { UA }\end{array}$

Contaminants

BVDV2 CPA

BVDV2 CPAE

BVDV2 EBTr

BVDV2 HE727

BVDV2 MMR-T

BVDV2 Parvo

BVDV2 Rubella

AU UG CU

AA UACC U - AU UG

AA UACC U - AU UG

AAUACC U - AU UUG

AA UACC U - AU UG

AAUACC U - AU UG

UAUGCU G - AU

$\begin{array}{ll}\text { UA } & \text { AU } \\ \text { UA } & \text { AU } \\ \text { UA } & \text { AU } \\ \text { UA } & \text { AU } \\ \text { UA } & \text { AU } \\ & \\ \text { UG } & \text { GC } \\ \text { UG } & G C \\ \text { UG } & G C \\ \text { UG } & G C \\ \text { JG } & G C \\ \text { UG } & G G \\ \text { JG } & G C \\ & G C\end{array}$

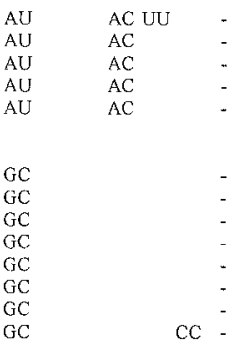

UK

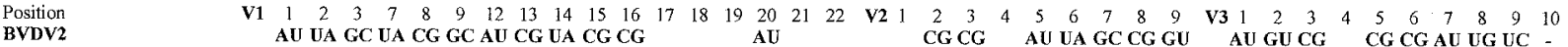

2a 13 similar with Belgian strain BSE921 divergent V1/17,20

BVDV2 167237

BVDV2 168149

$\begin{array}{ll}\text { BVDV2 } & 168149 \\ \text { BVDV2 } & 173157\end{array}$

$\begin{array}{ll}\text { BVDV2 } & 173157 \\ \text { BVDV2 } & 175375\end{array}$

BVDV2 Lees

GU

$\begin{array}{lllll}\text { AU UA CC } & \text { U }- & \text { AU } & \text { UG } & \text { GC } \\ \text { AU UA CC } & U- & \text { AU } & \text { UG } & \text { GC } \\ \text { AU UA CC } & \text { U }- & \text { AU } & \text { UG } & \text { GC } \\ \text { AU UA CC } & \text { U }- & \text { AU } & \text { UG } & \text { GC } \\ \text { AU UA CC } & \text { U }- & \text { AU } & \text { UG } & \text { GC }\end{array}$

$G C$
$G C$
$G C$
$G C$
$G C$ 


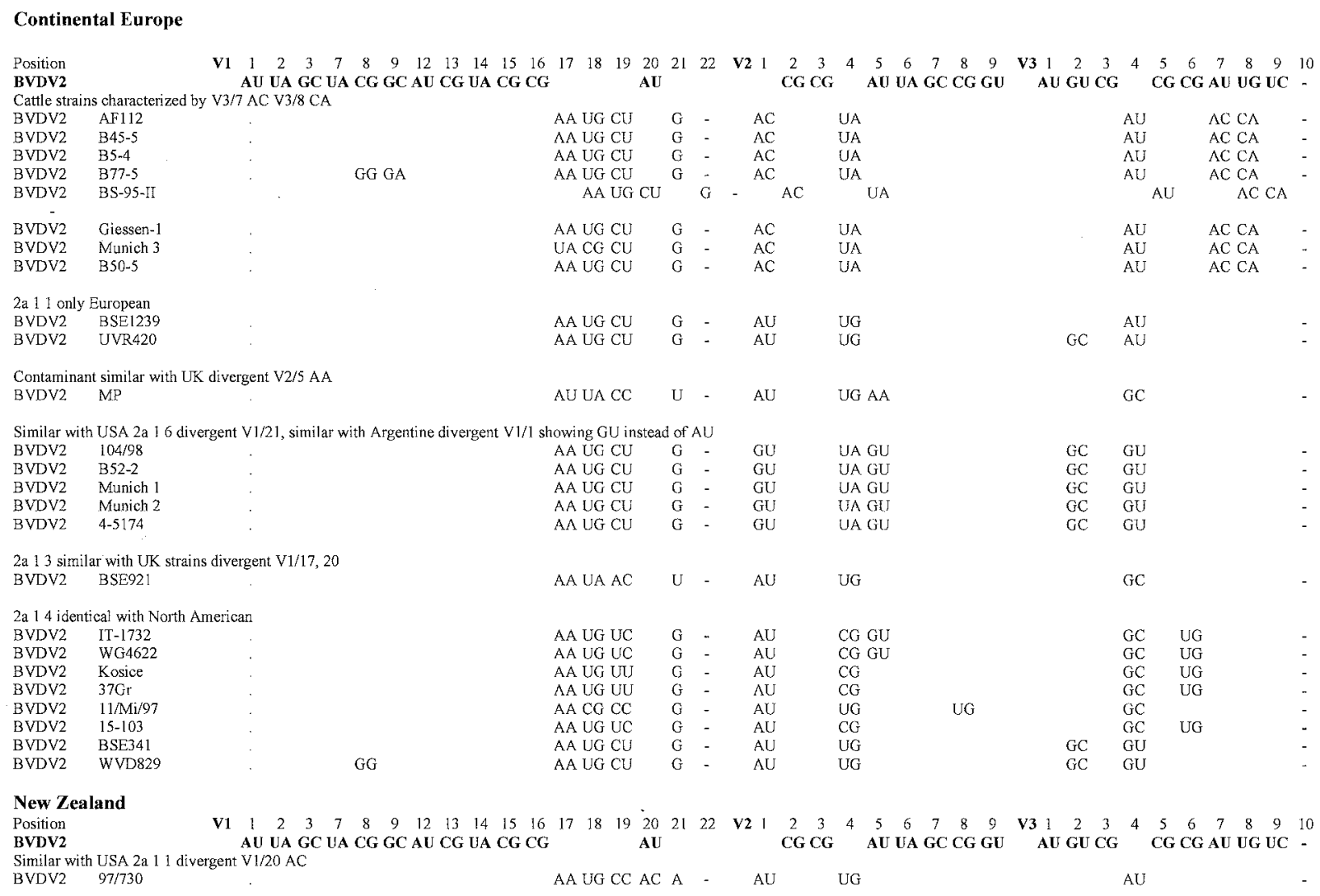

Fig. 1. BVDV2 palindromic secondary structure sequences and relation with geographical origin. Sequences are segregated according to geographic relevant groups. Specific characters on originality, shared sequences or variations are presented.

tal Europe, UK, New Zealand and Japan. Fied cattle strains from continental Europe, Japan and U.S.A., characterized by V3/7 AC and clustered in the same subgenotype 2 of genotype $2 \mathrm{a}$, were divergent in V3/8: German isolates showed CA, Japanes strains were characterized by UG or UU, strain from U.S.A. showed UA. Genomic variation could distinguish also strains isolated from Brazil and from Argentina. Within genotype $2 b$, Argentinean strains were divergent from Brazilian ones at the level of the V2 locus in position 1 showing GU and AU, respectively. Argentinean strains belonging to genotypes $2 \mathrm{~b}$ and $2 \mathrm{~d}$ were characterized by $\mathrm{V} 1 /$ 20 AA. Despite similarity within genotype 2a, subgenotype 1 , variant 6 , Argentinean strains showed specific nucleotide changes from other strains from Europe and U.S.A., clustered in the same variant. Argentinean cattle strains were similar with U.S.A. 2a 16 divergent V1/21, and similar with European divergent V1/1 showing GU instead of AU pairing. Similarly, strains affecting sheep showed divergence from those isolated from cattle. They correspond to variants 2 and 3 of subgenotype 1 within the genotype $2 \mathrm{a}$. The only strain affecting cattle showing relation being included in variant 13 were divergent in position 19 of V1. Among sequences originated from sheep, those from UK were characterised by AU pairing in position 17, UA in position 18 and $\mathrm{U}$ in position 20 in the V1 locus. Those from U.S.A. showed a characteristic CA bulge in position 6 of V2 locus. Contamination of biological products was related to genotypes $2 \mathrm{a}$ and $2 \mathrm{~d}$.

Correlation between clinical features related with isolation of BVDV-2 strains with genetic variation indicated that genotype $2 \mathrm{a}$; subgenotype 1 , variants 4 and 5 were related with hemorrhagic syndrome, and apparently as exception also variant 6 (Table 3 ). Within strains affecting cattle, highly virulent ones, exception made for 2 strains (AZ Spl and CD87), they were characterised by V1/19 UU or UC and V2/4 CG (stronger binding than UG detected in all low virulent strains). Similarly, ovine isolates from U.S.A. showed the same characteristic. Ovine strains related with border disease from UK showed characteristic UA pairing in $\mathrm{V} 1 / 18$ and $U$ in $\mathrm{V} 1 / 21$, shared by only one low virulent cattle strain. Low virulent cattle strains sequences showed in V1/19 CC, CU, AC, CA bulges or CG pairing, and in V2/ $4 \mathrm{UG}$ or UA.

The genotype BVDV-2a subgenotype 1 variant 2, 4 and 5 strains, including the hyper-virulent strain 890 (variant 5), presented mainly uracil as N1 and cytosine as N2 (UC), and all were related to high virulence (hemorrhagic syndrome or acute BVD). Within variant 4 , six strains presented the $\mathrm{CU}$ combination, but only one was related with high virulence as hemorrhagic syndrome. In the genotype BVDV-2a sub- 
Table 3. Summary of correlation between clinical features related with isolation of BVDV-2 strains with genetic variation

\begin{tabular}{|c|c|c|c|c|c|}
\hline \multicolumn{6}{|c|}{ Highly virulent strains } \\
\hline Genotype & Subgenotype & Variant & Host & Clinical presentation & umber of observations \\
\hline BVDV-2a & 1 & 2 & Sheep & Border disease & 2 \\
\hline BVDV-2a & 1 & 3 & Sheep & Border disease & 5 \\
\hline BVDV-2a & 1 & 4 & Cattle & Hemorrhagic syndrome & 11 \\
\hline BVDV-2a & 1 & 5 & Cattle & Hemorrhagic syndrome & 1 \\
\hline BVDV-2a & 1 & 6 & Cattle & Hemorrhagic syndrome & 1 \\
\hline \multicolumn{6}{|c|}{ Mucosal disease } \\
\hline BVDV-2a & 2 & - & Cattle & Fatal mucosal disease & 1 \\
\hline BVDV-2b & 2 & - & Cattle & Mucosal disease & 1 \\
\hline \multicolumn{6}{|c|}{ Low virulent strains } \\
\hline Genotype & Subgenotype & Variant & Host & Clinical presentation & \\
\hline BVDV-2a & 1 & 1 & Cattle & Fetal abortion & 5 \\
\hline BVDV-2a & 1 & 3 & Cattle & Neurological symptoms & 1 \\
\hline BVDV-2a & 1 & 4 & Cattle & Neurological symptoms & 3 \\
\hline BVDV-2a & 1 & 6 & Cattle & Mild symptoms & 7 \\
\hline BVDV-2a & 2 & - & Cattle & Respiratory symptoms, abortion & 12 \\
\hline BVDV-2b & 1 & $1,2,3,4$ & Cattle & Mild symptoms & 6 \\
\hline BVDV-2c & - & - & Cattle & Mild symptoms & 1 \\
\hline
\end{tabular}

genotype 1 variants 1 and 6 was evident only the combination of cytosine as N1 and uracil as N2 (CU) which was associated mainly low virulence as abortion, respiratory or neurological symptoms or persistent infection, with no lethal issue, exception made in strain CD87, associated to hemorrhagic syndrome. Similarly, in genotype BVDV-2a subgenotype 2, CU was generally associated with mild clinical symptoms, apart for strain Giessen-1, associated fatal mucosal disease. The genotype BVDV-2a subgenotype 1 variant 3 presented cytosine as N1 and uracil as N2 (CU) in relation with high virulence as border disease in sheep isolates as well as with mild clinical symptoms in one cattle isolate, and in contaminants. Furthermore, one BVDV-2a strain presented adenine as N1 and uracil as N2 (AU) related with low virulence as neurological symptoms. South American isolates belonging to genotypes BVDV2-b, BVDV2-c and BVDV2-d showed CU combination in relation with mild clinical course or contamination (genotype 2c), apart subgenotype 2 of BVDV2-b related with MD.

In strains isolated from sheep, all animals with symptoms of border disease, the presence of cytosine in position N1 and uracil in position N2, was the most frequent, in 5 out of 7 strains, Lees, 167 237, 168 149, 173157 and 175375 (all strains originated from UK). The presence of uracil in position $\mathrm{N} 1$ and cytosine in position N2 was observed in the two North American strains BD-78 and C413. In BVDV-2 strains adventitious contaminants of biological products, the presence of uracil in position $\mathrm{N} 1$ and cytosine in position $\mathrm{N} 2$ was observed in only two strains, the high virulence related strains WG4622 and IT-1732. All other tested strains, cell lines and biological products contaminants, with unknown virulence degree, showed cytosine in position N1 and uracil in position $\mathrm{N} 2$.

\section{DISCUSSION}

The observation made on the nucleotide sequences of the three variable loci at the level of the 5'-UTR genomic region of BVDV-2 Pestivirus strains allowed to the identification of consensus motifs shared by all the Pestivirus species. The BVDV-2 strains could be clearly differenced genetically, in terms of specific nucleotide substitutions, by the BVDV1 species. Four genotypes, BVDV-2a, BVDV-2b, BVDV-2c, and BVDV-2d, were classified within BVDV-2 by the PNS method. South American isolates represented three well distinct clusters among strains in the species. Relevant nucleotide variations in the secondary structure were evident at the level of the V1 palindromic locus. Genotypes BVDV-2c and BVDV-2d were restricted to Argentina. BVDV-2b was present in Brazil and Argentina, suggesting a link with animal exchange, even if the observation was made on a very limited number of available strains. However, between 1980 and 2002, limited bovine imports from Brasil were recorded ( 1 breeder in 1986, 1 breeder in 1988, 2 breeders in 1989, 1 breeder in 1990, 326 fattening cattle in 1998 and 211 in 1999) (National Animal Health Service, Buenos Aires, Argentina). Importation of bubalines has been also recorded (154 in 1998, 432 in 1999), but no sheep imports. In the same period, the number animals imported from Uruguay was higher (around 150,000 heads for slaughtering, and 5,000 breeders). Despite absence of epidemiological data, this could suggest an eventual indirect sorce of virus diffusion in the region through a neighbouring contry which is geographically situated between Brazil and 
Argentina.

Nomenclature for Pestivirus species is predominantly dependent on the animal host species from which they were isolated. There is extensive antigenic cross-reactivity among the species, and, they can cross the host species barrier and infect various animal species. The application of genetic analyses appeared necessary as most appropriate approach for differential diagnostic in order to solve such cross-infections which may obscure the rationale for the definition of the Pestivirus species according to their animal host. In the present study, eight BVDV-2 strains were ovine isolates. The sequence analysis showed that only BVDV-2a was related to virus circulation in sheep, whereas genotypes BVDV-2c, and BVDV-2d were prevalent in cattle. Strains have been isolated from animals suffering of border disease, similar symptoms caused with BDV, thus rising confusion in diagnosis. Among BDV strains previously classified depending on the host animal species, occurrence of other Pestivirus species has long been suspected [7, 10, 29]. Technical terms "true" BDV and "atypical" BDV have been used in order to discriminate authentic BDV strains from false BDV strains by Thiel and his colleagues $[1,3,4]$. This confusion was further examined by phylogenetic analysis of the E2 (gp53) region, Npro gene or 5'-UTR of ovine pestiviruses, and the BDV strains previously classified have been shown to include BVDV [3, 34, 38]. In a recent study, by using the PNS genotyping procedure, ovine isolates showed palindromic structure in the 5'-UTR characteristic to the BVDV-1a and BVDV-1b genotypes, and to BVDV-2 [15]. The number of BVDV-2 ovine isolates, reported until now, is limited. In addition, it is not excluded that the five ovine strains reported by Vilcek et al. [38] are contaminants from fetal calf serum during cultivation of cells [37]. Therefore, particular attention is required in performing isolation on cell. RT-PCR assay for the rapid recognition of virus in blood sample or original tissue homogenates with no cultivation on cells for border disease clinical cases could be a useful approach.

The two nucleotides, related to virulence degree in Pestivirus strains, were identifiable at the palindromic structures, variable loci V1 loop and V2 stem regions in the IRES in the 5'-UTR of the genomic RNA. Different nucleotide combinations could be identified. The most frequent was $\mathrm{CU}$. UC and AU pairings were less frequent. The two nucleotide combinations UC and CU resulted associated to high virulence and in relation with genotype BVDV-2a. The nucleotide combination CU was shared with low virulence strains and in relation to genotypes BVDV-2a, 2b and 2c. In cattle, the presence of uracil in position N1 and cytosine in position $\mathrm{N} 2$ was observed in 11 strains related to severe clinical disease. The presence of cytosine in position $\mathrm{N} 1$ and uracil in position N2 was observed in 39 strains, exception made for 2 strains, mainly related to mild forms of clinical disease. The presence of cytosine at position N1 and uracil at position N2 was identified in two field isolates related to hemorrhagic syndrome from North America. In one strain was observed adenine in position N1 and uracil in position N2. The observation made by Topliff and Kelling [35] could be partially confirmed in our study. Out of the 13 tested cattle strains related to high virulence, the $84.61 \%$ showed the suggested virulence marker UC. However, the strains 890 and CD87 had to be considered of high virulence and low virulence, respectively, giving that the strain 890 presented uracil in position $\mathrm{N} 1$ and cytosine in position N2, and the strain CD87 showed the 2 nucleotides in the inverted locations, with cytosine at position $\mathrm{N} 1$ and uracil at position N2. Both strains were reported by Pellerin et al. [25] from outbreaks in Canadian cattle, thus, equally responsible of haemorrhagic syndrome. Similarly, the strain AZ Spl, responsible of haemorrhagic syndrome in U.S.A., both showed N1 C and N2 U, the alleged low virulence related nucleotide combination.

In sheep, BVDV-2 was responsible for severe illness irrespective for the presence of UC or CU combination, indicating the absence of direct relationship with clinical manifestation of Border disease. However, the presence of uracil in position $\mathrm{N} 1$ and cytosine in position $\mathrm{N} 2$ was observed in the 2 North American isolates. Cytosine in position $\mathrm{N} 1$ and uracil in position $\mathrm{N} 2$ was detected in the five strains from UK, which have been suspected contaminants [38].

It seems appropriate to refer to the suggested nucleotide combination as virulence marker. In the case of the iatrogenic accident occurred in the Netherlands among cattle, who received a live virus vaccine contaminated with pestivirus BVDV-2, genetic investigations performed by State Institutes indicated two specific nucleotide substitutions in the 5'-UTR as characteristic of highly virulent BVDV-2, according to Topliff and Kelling [11]. However, this could raise the risk of underestimation for other nucleotide combinations potentially related to high virulence.

The observation of Pilipenko on the poliovirus 5'-UTR demonstrated important aspects; useful base for investigations on virulence and the study of Topliff and Kelling revealed similar relationship between virulence characteristic and the IRES of BVDV-2. However, increasing the number of evaluated strains, it appeared that the virulence nucleotide markers are more complex and the definition of virulence activity among BVDV-2 species, in terms of nucleotide sequence, remains still undefined. Furthermore, it is unclear the high variability in the other Pestivirus species, despite the genomic similarity to poliovirus, as for BVDV-2. In conclusion, uracil in position N1 and cytosine in position N2 appeared to be related to high virulencein the BVDV-2 species, but it was not always determinative for virulence markers since high virulence was also associated to cytosine in positions $\mathrm{N} 1$ and $\mathrm{N} 2$ and cytosine in position $\mathrm{N} 1$ and uracil in position N2.

The palindromic loci represented, with 77-85 nucleotides, a very limited portion of the virus genome. Within these short sequences, it was sufficient the evaluation of only 18 nucleotides to obtain with certitude the characterization of the genus. Species were characterized through the evaluation of only 2 to 8 nucleotides. Similarly, the geno- 
type was defined with only 2 to 6 nucleotides. These peculiar aspects resumed the high specificity of the PNS method and the reliability of the provided results. The palindromic nucleotide substitution analysis in the 5'-UTR demonstrated a rationale and simple approach for viral investigations. Secondary structures predicted at the three variable regions in the 5'-UTR showed typical PNS which were useful for classification or genotyping of Pestivirus. Due to the worldwide economic importance of these viruses, and the difficulties encountered in the control of the diseases, it is, therefore, important to understand the genetic aspects of the viruses and their evolutionary history. These observations suggest that evaluation of genomic secondary structure, by identifying markers of virus genomic characteristics in relation with environment-geographic segregation as well as animal host - and clinical course, may be applied as useful tool for epidemiological evaluation, improving our understanding of the BVDV-2 species epizootiology, and possibly for other species of the genus Pestivirus.

ACKNOWLEDGMENTS. We express our thanks for excellent technical assistance to Mrs F. Maillet, Veterinary Clininc Saint François, Ambilly, France, and Dr L. Jones, Instituto de Virologia, Centro de Investigacion en Ciencias Veterinarias y Agronomicas (CICVyA), INTA-Castelar, Castelar, Buenos Aires, Argentina.

\section{REFERENCES}

1. Becher, P., König, M., Paton, D.J. and Thiel, H.-J. 1995. Further characterization of border Disease virus isolates: evidence for the presence of more than three species within the genus pestivirus. Virology 209: 200-206.

2. Becher, P., Orlich, M., König, M. and Thiel, H.J. 1999. Nonhomologous RNA recombination in bovine viral diarrhea virus: molecular characterization of a variety of subgenomic RNAs isolated during an outbreak of fatal mucosal disease. J. Virol. 73: 5646-5653.

3. Becher, P., Orlich, M., Shannon, A.D., Hornet, G., Koning, M. and Thiel, H.J. 1997. Phylogenetic analysis of pestivirus from domestic and wild ruminants. J. Gen. Virol. 78: 1357-1366.

4. Becher, P., Shannon, A.D., Tautz, N. and Thiel, H.-J. 1994. Molecular characterization of border disease virus, a pestivirus from sheep. Virology 198: 542-551.

5. Bolin, S.R. and Ridpath, J.F. 1992. Differences in virulence between two noncytopathic bovine viral diarrhea viruses in calves. Am. J. Vet. Res. 53: 2157-2163.

6. Broes, A., Wellemans, G. and Dheedene, J., 1992. Syndrome hémorrhagique chez des bovins infectés par le virus de la diarrhée virale bovine (BVD/MD). Ann. Méd. Vét. 137: 33-38.

7. Carlsson, U. 1991. Border disease in sheep caused by transmission of virus from cattle persistently infected with bovine virus diarrhea virus. Vet. Rec. 128: 145-147.

8. Couvreur, B., Letellier, C., Collard, A., Quenon, P., Dehan, P., Hamers, C., Pastoret, P. P. and Kerkhofs P. 2002. Genetic and antigenic variability in bovine viral diarrhea virus (BVDV) isolates from Belgium. Virus Res. 85: 17-28.

9. Deng, R. and Brock, K.V. 1992. Molecular cloning and nucleotide sequence of the pestivirus genome, non-cytopathic bovine viral diarrhea virus strain SD-1. Virology 191: 867-879.
10. Edwards, S., Roehe, P.M. and Ibata, G. 1995. Comparative studies of border disease and closely related virus infections in experimental pigs and sheep. Brit. Vet. J. 151: 181-188.

11. Falcone, E., Tollis, M. and Conti, G. 2000. Bovine Viral Diarrhea disease associated with a contaminated vaccine. Vaccine 18: $387-388$

12. Fauquet, C.M., Mayo, M.A., Maniiloff, J., Desselberger, U. and Ball, L.A. 2005. Virus Taxonomy. Classification and Nomenclature of Viruses. Elsevier, Academic Press, San Diego.

13. Flores, E.F., Ridpath, J.F., Weiblen, R., Vogel, F.S.F. and Gil, L.H.V.G. 2002. Phylogenetic analysis of Brazilian bovine viral diarrhea virus type 2 (BVDV-2) isolates: evidence for a subgenotype whitin BVDV-2. Virus Res. 87: 51-60.

14. Freier, S.M., Kierzek, R., Jaege, J.A., Sugimoto, N., Caruthers, M.H., Nielson, T. and Turner, D.H. 1986. Improved freeenergy parameters for predictions of RNA duplex stability. Proc. Natl. Acad. Sci. U.S.A. 83: 9373-9377.

15. Giangaspero, M. and Harasawa, R. 2002. Eterogeneità dei Pestivirus ovini. Vet. Ital. 38: 60-65.

16. Harasawa, R. 1994. Comparative analysis of the 5' non-coding region of pestivirus RNA detected from live virus vaccines. $J$. Vet. Med. Sci. 56: 961-964.

17. Harasawa, R. and Giangaspero, M. 1998. A novel method for pestivirus genotyping based on palindromin nucleotide substitutions in the 5'-untranslated region. J. Virol. Methods 70: 225-230.

18. Harasawa, R., Hikiji, K., Tanabe, H., Takada, Y. and Mizusawa, H. 1993. Detection of adventitious pestivirus in cell cultures by polymerase chain reaction using nested-pair primers. Tissue Cult. Res. Commun. 12: 215-220.

19. Harasawa, R. and Sasaki, T. 1995. Sequence analysis of the 5' untranslated region of pestivirus RNA demonstrated in interferons for human use. Biologicals 23: 263-269.

20. Harasawa, R. and Tomiyama, T. 1994. Evidence of pestivirus RNA in human virus vaccines. J. Clin. Microbiol. 32: 1604 1605.

21. Jones, L.R., Zandomeni, R.O. and Weber, E.L. 2001. Genetic typing of bovine viral diarrhea virus isolates from Argentina. Vet. Microbiol. 81: 367-375.

22. Luzzago, C., Zecconi, A., Bronzo, V., Bazzocchi, C., Ruggeri, A. and Ruffo, G. 2000. Bovine viral diarrhea virus genotype I and II in Italian dairy herd in 1995. pp. 397-398. In: Proceedings of the 5th International Congress of the European Society for Veterinary Virology "Veterinary virology in the new millennium", August 26-30 2000, Brescia, Italy.

23. Nagai, M., Ito, T., Sugita, S., Genno, A., Takeuchi, K., Ozawa, T., Sakoda, Y., Nishimori, T., Takamura, K. and Akashi, H. 2001. Genomic and serological diversity of bovine viral diarrhea virus in Japan. Arch. Virol. 146: 685-696.

24. Nagai, M., Sato, M., Nagano, H., Pang, H., Kong, X., Murakami, T., Ozawa, T. and Akashi, H. 1998. Nucleotide sequence homology to bovine viral diarrhea virus 2 (BVDV 2) in the 5' untranslated region of BVDVs from cattle with mucosal disease or persistent infection in Japan. Vet. Microbiol. 60: 271-276.

25. Pellerin, C., van den Hurk, J., Lecomte, J. and Tussen, P. 1994. Identification of a new group of bovine diarrhea virus strains associated with severe outbreaks and high mortalities. Virology 203: 260-268.

26. Pilipenko, E.V., Gmyl, A.P., Maslova, S.V., Svitkin, Y.V., Sinyakov, A.N. and Agol, V.I. 1992. Prokariotic-like Cis Elements in the Cap-Indipendent Internal Initiation of Translation 
on Picornavirus RNA. Cell 68: 119-131.

27. Rebhun, W.C., French, T.W., Perdrizet, J.A., Dubovi, E.J., Dill, S.G. and Karcher, L.F. 1989. Thrombocytopenia associated with acute bovine virus diarrhea infection in cattle. J. Vet. Intern. Med. 3: 42-60.

28. Ridpath, J.F., Bolin, S.R. and Dubovi, E.J. 1994 Segregation of Bovine Viral Diarrhoea virus into genotypes. Virology 205: 66-74.

29. Roehe, P.M., Woodward, M.J. and Edwards, S. 1992. Characterization of p20 gene sequences from a border disease like pestivirus isolated from pigs. Vet. Microbiol. 33: 231-238.

30. Sakoda, Y., Ozawa, S., Damrongwatanapokin, S., Sato, M., Ishikawa, K. and Fukusho, A. 1999. Genetic heterogeneity of porcine and ruminant pestiviruses mainly isolated in Japan. Vet. Microbiol. 65: 75-86.

31. Sullivan, D.G., Chang, G.J., Trent, D.W. and Akkina, R.K. 1994. Nucleotide sequence analysis of the structural gene coding region of the pestivirus border disease virus. Virus Res. 33: 219-228.

32. Tajima, M., Frey, H.R., Yamato, O., Maede, Y., Moenning, V. Scholz, H. and Greiser-Wilke, I. 2001. Prevalence of genotypes 1 and 2 of bovine viral diarrhea virus in Lower Saxony, Germany. Virus Res. 76: 31-42.

33. Thiel, W. 1993. Kasuitischer beitrag zu hämorrhagischen diathesen bei kälbern mit BVD virusinfektion. Tierärz. Praxis
21: 413-416.

34. Tijssen, P., Pellerin, C., Lecomte, J. and Van den Hurk, J. 1996. Immunodominant E2 (gp53) sequences of highly virulent bovine viral diarrhea group II viruses indicate a close resemblance to a subgroup of border disease viruses. Virology 217: $356-361$.

35. Topliff, C. L. and Kelling, C.L. 1998. Virulence markers in the 5 ' untranslated region of genotype 2 bovine viral diarrhea virus isolates. Virology 250: 164-172.

36. Vilček, O.E., Durkovic, B., Bobakova, M., Sharp, G. and Paton, D.J. 2002. Identification of bovine viral diarrhoea virus 2 in cattle in Slovakia. Vet. Rec. 151: 150-152.

37. Vilcek, O.E. and Paton, D.J. 2000. A RT-PCR assay for the rapid recognition of border disease virus. Vet. Res. 31: 435.

38. Vilček, S., Nettleton, P.F., Paton, D.J. and Belak, S. 1997. Molecular characterization of ovine pestiviruses. J. Gen. Virol. 78: 725-735.

39. Vilcek, S., Paton, D.J., Durkovic, B., Strojny, L., Ibata, G., Moussa, A., Loitsch, A., Rossmanith, W., Vega, S., Scicluna, M.T. and Palfi, V. 2001. Bovine viral diarrhoea virus genotype 1 can be separated into at least eleven genetic groups. Arch. Virol. 146: 99-115.

40. Zuker, M. and Stiegler, P. 1981. Optimal computer folding of large RNA sequences using thermodynamics and auxiliary. Nucleic Acids Res. 9: 133-148. 